\title{
Critical temperature in weakly interacting multicomponent field theory
}

\author{
V.I. Yukalov ${ }^{1, \star}$ and E.P. Yukalova ${ }^{2, \star \star}$ \\ ${ }^{1}$ Bogolubov Laboratory of Theoretical Physics, \\ Joint Institute for Nuclear Research, Dubna 141980, Russia \\ ${ }^{2}$ Laboratory of Information Technologies, \\ Joint Institute for Nuclear Research, Dubna 141980, Russia
}

\begin{abstract}
A method is suggested for calculating the critical temperature in multicomponent field theory with weak interactions. The method is based on self-similar approximation theory allowing for the extrapolation of series in powers of asymptotically small coupling to finite and even infinite couplings. The extrapolation for the critical temperature employs self-similar factor approximants. The found results are in perfect agreement with Monte Carlo simulations.
\end{abstract}

\section{Introduction}

Nuclear matter under extreme conditions, when varying baryon density or temperature, can experience different phase transitions [1-7]. The first thing one needs in studying a phase transition is to find out the related transition point, which is not always an easy task. In the present report, we explain typical problems arising in calculating transition points by the example of an effective multicomponent field theory. The critical temperature in this theory, being calculated by means of loop expansions, leads to a series whose terms are divergent. So that one has to sum a series of divergent terms, while the resulting temperature is, of course, finite. We suggest a method for overcoming this difficulty and illustrate it by calculating the critical temperature of an $N$-component field theory for different $N$. Comparing our approach with Monte Carlo simulations, when these are available, we find that our method provides the results that are in beautiful agreement with the Monte Carlo data.

We emphasize that our aim in this report is not to consider a particular phase transition that could occur in nuclear matter under varying parameters, but to develop a general strategy that would be valid for calculating critical temperatures in any of phase transitions of second-order type. As examples of such critical phase transitions in nuclear matter, occurring under varying barion density or temperature, it is possible to mention the transition at the critical point between hadron gas and hadron liquid, the transitions between hadron liquid and nuclear superfluid, between quark-gluon plasma and colour superconductor, Bose condensation of multiquark clusters, possible transitions between gluon and gluball phases, and some scenarios of deconfinement transition [1-7]. In the present report, we treat

\footnotetext{
${ }^{\star}$ e-mail: yukalov@theor.jinr.ru

$\star \star$ e-mail: yukalova@theor.jinr.ru
} 
a model possessing those problems that are typical in calculating critical temperatures in practically any continuous phase transition.

In what follows, we employ the system of units, where the Planck and Boltzmann constants are set to unity.

We start with a nonrelativistic $3+1$ - dimensional field theory with the action

$$
S[\psi]=\int L[\psi] d t, \quad L[\psi]=\int \psi^{\dagger} i \frac{\partial}{\partial t} \psi d x-H[\psi]
$$

whose Lagrangian contains the energy part

$$
H[\psi]=\int\left[\psi^{\dagger}\left(-\frac{\nabla^{2}}{2 m}-\mu\right) \psi+\frac{\lambda}{2}|\psi|^{4}\right] d x,
$$

with the coupling parameter

$$
\lambda=4 \pi \frac{a_{s}}{m}
$$

expressed through the scattering length $a_{s}$. The term $|\psi|^{4}$ is understood as $\left(\psi^{\dagger} \psi\right)^{2}$. In the threedimensional space, the variable $x=\left\{x_{\alpha}: \alpha=1,2,3\right\}$.

Being interested in static quantities at finite temperature, we pass to the imaginary time formalism by accomplishing the Wick rotation [8]. Then the field $\psi$ is periodic with respect to the imaginary time, $\psi(x, 0)=\psi(x, \beta)$, where $\beta \equiv 1 / T$ is inverse temperature. Due to this periodicity, the field $\psi$ may be decomposed into imaginary-time frequency modes with Matsubara frequencies $2 \pi T j$, where the integer $j=0, \pm 1, \pm 2, \ldots$. Near the transition point, the main contribution to the partition function is played by the zero Matsubara mode, while the nonzero Matsubara frequencies can be integrated out. To take into account $N$ internal degrees of freedom, the term $|\psi|^{2}$ is interpreted as $\sum_{n=1}^{N} \varphi_{n}^{2}$, with the field $\varphi_{n}$ for each of the components. In this way, we come to the partition function being the trace of the Boltzmann weight $\exp S[\varphi]$, with the effective action

$$
S[\varphi]=\int\left[\frac{1}{2}\left(\frac{\partial \varphi}{\partial x}\right)^{2}+\frac{m_{e f f}^{2}}{2} \varphi^{2}+\frac{\lambda_{e f f}}{4 !} \varphi^{4}\right] d x,
$$

corresponding to an $\mathrm{N}$-component field theory, with the effective parameters

$$
m_{e f f}^{2}=-2 m T \mu, \quad \lambda_{e f f}=48 \pi m T a_{s} .
$$

The details of the above procedure are well known and were described in many publications, e.g., in Refs. [8-12].

Thus, we need to study phase transitions in the $N$-component filed theory with action (4). This action represents a wide class of physical systems, depending on the number of components. For instance, $N=0$ corresponds to dilute polymer solutions, $N=1$, to the Ising-type models, $N=2$, to the XY model and superfluids, and $N=3$ corresponds to the Heisenberg model.

\section{Phase transition temperature}

Action (4) describes the $O(N)$-symmetric multicomponent filed theory with the $N$-component field $\varphi=\left\{\varphi_{n}(x): n=1,2, \ldots, N\right\}$. The action is invariant under the inversion symmetry

$$
\varphi_{n} \rightarrow-\varphi_{n} \quad(n=1,2, \ldots, N)
$$


This implies that one of the solutions for the order parameter $\eta \equiv\langle\varphi\rangle$, which is the average of the field, is zero, $\langle\varphi\rangle=0$.

But below the critical temperature $T_{c}$, the symmetry can become spontaneously broken, when a nonzero order parameter $\langle\varphi\rangle$ provides a lower free energy $F=-T \ln \operatorname{Tr} \exp (-S[\varphi])$, so that

$$
F(\eta \neq 0)<F(\eta \equiv 0)
$$

One says that at the critical temperature $T_{c}$ there occurs a phase transition, where the order parameter changes from zero to a nonzero value:

$$
\eta \equiv 0 \quad\left(T>T_{c}\right) ; \quad \eta \neq 0 \quad\left(T<T_{c}\right) .
$$

In the considered case, the order parameter at $T_{c}$ is continuous, which signifies the second order phase transition.

The transition temperature depends on the interaction strength that can be characterized by the gas parameter

$$
\gamma \equiv \rho^{1 / 3} a_{s},
$$

in which $\rho$ is a particle density and the scattering length is assumed to be positive, $a_{s}>0$. For the ideal gas, with no interactions, the critical temperature is

$$
T_{0} \equiv T_{c}(0)=\frac{2 \pi}{m}\left[\frac{\rho}{\zeta(3 / 2)}\right]^{2 / 3},
$$

which is evident from (1) and (2) under $\lambda=0$. The problem is to find the dependence of the critical temperature $T_{c}(\gamma)$ on the gas parameter at small values of the latter.

One defines the relative critical temperature shift

$$
\frac{\Delta T_{c}}{T_{0}} \equiv \frac{T_{c}-T_{0}}{T_{0}}
$$

which, at weak interactions, is linear in the gas parameter:

$$
\frac{\Delta T_{c}}{T_{0}} \simeq c_{1} \gamma \quad(\gamma \rightarrow 0)
$$

as it has been proved in Refs. [9-12]. The difficulty in finding the coefficient $c_{1}$ is in the impossibility of employing perturbation theory with respect to $\gamma$, which, as explained below, looses its sense at the point $T_{c}$. Various ways of solving this problem have been reviewed in [13, 14].

One is able to calculate the shift coefficient $c_{1}$ involving the loop expansion [15], which yields [16-18] an expansion in powers of the variable

$$
x=(N+2) \frac{\lambda_{e f f}}{\sqrt{\mu_{e f f}}},
$$

in which $N$ is the number of the components, $\lambda_{e f f}$ is an effective coupling, defined in (5), and $\mu_{e f f}$ is

$$
\mu_{e f f}=m_{e f f}^{2}-\Sigma(0,0),
$$

with $\Sigma(0,0)$ being the self-energy at zero limit of energy and momentum. 
In seven loops, one comes to the asymptotic expansion

$$
c_{1}(x) \simeq \sum_{n=1}^{5} a_{n} x^{n} \quad(x \rightarrow 0) .
$$

The problem arises, when one considers the above expansion at the critical temperature $T_{c}$, where $\mu_{\text {eff }}$ becomes zero, because of which the expansion variable tends to infinity, $x \rightarrow \infty$. Since this expansion has been derived for asymptotically small $x$, the tendency of the latter to infinity makes the expansion senseless. To make sense of such a difficulty, it is necessary to invent a way of finding an effective sum of the asymptotic series, derived for $x \rightarrow 0$, which would extrapolate the sum to finite values of $x$ and including $x \rightarrow \infty$.

It is worth mentioning that the extrapolation of asymptotic series for a small variable to arbitrary values of the latter can be accomplished in the frame of optimized perturbation theory [19, 20]. Different variants of this theory have been employed for finding the shift coefficient $c_{1}$ by introducing control functions with a variable change $[17,18]$ or incorporating them into initial approximations [21-24]. However, the results of such calculations strongly depend on how the control functions are introduced, and also these calculations are rather cumbersome requiring the use of optimization conditions defining control functions at each approximation order.

Below we suggest a general uniquely defined method for extrapolating asymptotic series of a small variable to arbitrary values of the variable, including its infinite limit.

\section{Self-similar approximation theory}

A general method of extrapolation is based on self-similar approximation theory [25-28]. Although the final prescription that will be formulated below is rather simple, we briefly delineate the main steps of the theory to give the reader the impression of how the employed approximants have been obtained.

Self-similar approximation theory is based on the following important points. First, it is necessary to transform an initially given divergent sequence $\left\{f_{k}(x)\right\}$ of the series of order $k$ for the sought function into a convergent sequence $\left\{F_{k}\left(x, u_{k}(x)\right)\right\}$ by incorporating control functions $u_{k}(x)$. The latter can be introduced either in an initial approximation $f_{0}(x, u)$, or through a variable change $x=z(x, u)$, or by a sequence transformation $T(u) f_{k}(x)=F_{k}(x, u)$. A sequence is convergent if and only if, for a given positive $\varepsilon$, there exists $k_{\varepsilon}$ such that for all $k>k_{\varepsilon}$ the Cauchy criterion of convergence is valid:

$$
\left|F_{k+p}\left(x, u_{k+p}\right)-F_{k}\left(x, u_{k}\right)\right|<\varepsilon .
$$

Control functions can be defined by minimizing the Cauchy cost functional

$$
C[u]=\frac{1}{2} \sum_{k}\left|F_{k+p}\left(x, u_{k+p}\right)-F_{k}\left(x, u_{k}\right)\right|^{2}
$$

At the next step, we consider the passage from an approximation $F_{k}$ to $F_{k+1}$ and so on as the motion in discrete time $k$. To formulate this in terminology of dynamical theory, we impose the reonomic constraint

$$
F_{0}\left(x, u_{k}(x)\right)=f, \quad x=x_{k}(f)
$$

and introduce the endomorphism

$$
y_{k}(f) \equiv F_{k}\left(x_{k}(f), u_{k}\left(x_{k}(f)\right)\right)
$$


In order for the Cauchy cost functional (17) to have the absolute minimum zero, it is necessary that endomorphism (19) would satisfy the relation

$$
y_{k+p}(f)=y_{k}\left(y_{p}(f)\right)
$$

that is termed the property of functional self-similarity. Relation (20) defines a dynamical system in discrete time, that is, a cascade, whose trajectory is bijective to the approximation sequence $\left\{F_{k}\right\}$, because of which this dynamical system is called the approximation cascade.

It is more convenient to deal with a dynamical system in continuous time, i.e., a flow. For this purpose, it is possible to embed the approximation cascade into an approximation flow, which implies that the flow trajectory passes through all points of the cascade trajectory,

$$
\left\{y_{k}(f): k \in \mathbb{Z}_{+}\right\} \subset\left\{y_{t}(f): t \in \mathbb{R}_{+}\right\}
$$

For the approximation flow, the self-similarity relation (20) can be rewritten as the Lie equation

$$
\frac{\partial}{\partial t} y_{t}(f)=v\left(y_{t}(f)\right), \quad v(f) \equiv\left[\frac{\partial}{\partial t} y_{t}(f)\right]_{t=0} .
$$

The Lie equation of motion, being integrated, yields the evolution integral

$$
\int_{y_{k}}^{y_{k}^{*}} \frac{d y}{v_{k}(y)}=t_{k}
$$

in which $v_{k}$ is the cascade velocity that is the Euler discretization of the flow velocity, $y_{k}^{*}$ is a quasi-fixed point, and $t_{k}$ is an effective time required for reaching $y_{k}^{*}$ from $y_{k}$. The quasi-fixed point $y_{k}^{*}$ represents the effective limit of the flow trajectory, hence, being bijective to the approximation sequence through notation (19), it represents the self-similar approximation $f_{k}^{*}(x)$ for the sought function $f(x)$.

The stability of the approximation procedure can be controlled by studying the local map multipliers

$$
\mu_{k}(f) \equiv \frac{\delta y_{k}(f)}{\delta y_{k-1}(f)} .
$$

The motion is locally stable provided that the absolute values of the map multipliers are not larger than one. Approaching a fixed point, one has $\left|\mu_{k}(f)\right| \rightarrow 1$ as $y_{k} \rightarrow y^{*}$. More details on the self-similar approximation theory can be found in the papers [25-28] and summarized in the review papers [2931].

Let us concretize the approach by considering a function for which one can find only an asymptotic series at a small variable,

$$
f(x) \simeq f_{k}(x) \quad(x \rightarrow 0),
$$

with the $k$-th order expansion

$$
f_{k}(x)=f_{0}(x)\left(1+\sum_{n=1}^{k} a_{n} x^{n}\right),
$$

in which $f_{0}(x)$ is a known term. Applying a fractal transform [30] to series (26) and following the procedure delineated above, we come to the self-similar factor approximants

$$
f_{k}^{*}(x)=f_{0}(x) \prod_{i=1}^{N_{k}}\left(1+A_{i} x\right)^{n_{i}},
$$


where $N_{k}=k / 2$ for even $k=2,4, \ldots$ and $N_{k}=(k+1) / 2$ for odd $k=3,5, \ldots$. The parameters $A_{i}$ and $n_{i}$ are defined by the accuracy-through-order procedure $f_{k}^{*}(x) \simeq f_{k}(x)$ as $x \rightarrow 0$, with the scaling $A_{1}=1$ for odd approximants [32-35].

We aim at studying the limit of large $x$. Let the known term $f_{0}(x)$ behave as

$$
f_{0}(x) \simeq A x^{\alpha} \quad(x \rightarrow \infty) .
$$

If the large-variable limit of the sought function is

$$
f(x) \simeq B x^{\beta} \quad(x \rightarrow \infty),
$$

then the powers of approximant (27) have to obey the constraint

$$
\alpha+\sum_{i=1}^{N_{k}} n_{i}=\beta \quad(\beta \neq 0),
$$

which, for the case of a finite large-variable limit, reduces to

$$
\alpha+\sum_{i=1}^{N_{k}} n_{i}=0 \quad(\beta=0) .
$$

In this way, we get a sequence of self-similar factor approximants (27). If a number of terms in sum (26) are available, then all we need is to observe the numerical convergence of the sequence $\left\{f_{k}^{*}\right\}$. When there are a few of such terms, the convergence of self-similar approximants can be essentially accelerated by constructing a quadratic spline

$$
q(x, t)=b_{0}(x)+b_{1}(x) t+b_{2}(x) t^{2}
$$

whose coefficient functions are defined through the conditions

$$
q(x, 0)=f_{k-2}^{*}(x), \quad q(x, 1)=f_{k-1}^{*}(x), \quad q(x, 2)=f_{k}^{*}(x) .
$$

The self-similar approximant for this spline reads as

$$
q^{*}(x, t)=b_{0}(x)[1+A(x) t]^{n(x)} .
$$

The latter provides an extrapolation of the sequence $f_{k-2}^{*}, f_{k-1}^{*}$, and $f_{k}^{*}$ to larger orders by setting $t \geq 2$. The minimal extrapolation involves $t=2$ and $t=3$ yielding the doubly renormalized approximant

$$
f_{k}^{* *}(x)=\frac{1}{2}\left[q^{*}(x, 2)+q^{*}(x, 3)\right]
$$

whose error value is given by the difference of $q^{*}(x, 2)$ and $q^{*}(x, 3)$.

\section{Calculation of temperature shift}

We accomplish this procedure for the critical temperature shift (12) using the fifth-order series (15). The coefficients $a_{n}$, calculated using the data of the seven-loop expansion by Kastening $[17,18]$, for different numbers of the components $N$, are presented in Table 1. 
Note that in expansion (15) the variable $x$ is assumed to be small, such that $x \rightarrow 0$, independently of the values of the parameters entering notation (13). But at the end, we have to find the limit $x \rightarrow \infty$, because of which the final answer for the coefficient $c_{1}$ is $c_{1}=\lim _{x \rightarrow \infty} c_{1}^{* *}(x)$.

Following the scheme of the previous section, we find the $k$-order factor approximants $f_{k}(x)^{*}$ for the quantity $c_{1}(x)$ in third order,

$$
f_{3}^{*}(x)=a_{1} x(1+x)^{n_{1}}\left(1+A_{2} x\right)^{n_{2}}
$$

with $n_{1}+n_{2}=-1$, in fourth order,

$$
f_{4}^{*}(x)=a_{1} x\left(1+A_{1} x\right)^{n_{1}}\left(1+A_{2} x\right)^{n_{2}},
$$

where $n_{1}+n_{2}=-1$, and in fifth order,

$$
f_{5}^{*}(x)=a_{1} x(1+x)^{n_{1}}\left(1+A_{2} x\right)^{n_{2}}\left(1+A_{3} x\right)^{n_{3}},
$$

with $n_{1}+n_{2}+n_{3}=-1$.

Since finally we need the limits $x \rightarrow \infty$, we find from the above approximants the limits

$$
f_{k}^{*} \equiv \lim _{x \rightarrow \infty} f_{k}^{*}(x)
$$

in the forms

$$
f_{3}^{*}=a_{1} A_{2}^{n_{2}}, \quad f_{4}^{*}=a_{1} A_{1}^{n_{1}} A_{2}^{n_{2}}, \quad f_{5}^{*}=a_{1} A_{2}^{n_{2}} A_{3}^{n_{3}},
$$

whose values are given in Table 2. After taking the limit $x \rightarrow \infty$, the variable $x$ does not enter the expressions below.

Accelerating the convergence by means of splines, as is explained above, we construct the quadratic polynomials

$$
q(t)=b_{0}+b_{1} t+b_{2} t^{2},
$$

with the coefficients defined by the equations

$$
q(0)=f_{3}^{*}, \quad q(1)=f_{4}^{*}, \quad q(2)=f_{5}^{*},
$$

which are listed in Table 3. The factor approximant for the spline $q(t)$ reads as

$$
q^{*}(t)=b_{0}(1+A t)^{n},
$$

with the values $A$ and $n$ from Table 3 . The values

$$
q_{2}^{*} \equiv q^{*}(2), \quad q_{3}^{*} \equiv q^{*}(3)
$$

are presented in Table 2 .

Finally, the sought coefficient $c_{1}$, defined by formula (35), under $x \rightarrow \infty$ is given in Table 4 . We also compare our calculations for $c_{1}$ with the available Monte Carlo simulations [36-39], as well as with the results obtained by optimized perturbation theory (OPT) $[17,18]$. As it is seen, the values of $c_{1}$, derived in our approach, are in perfect agreement with the Monte Carlo simulations. The advantage of the self-similar factor approximants, we employed, is that they are uniquely defined and do not involve the use of control functions at the final step. While the results of optimized perturbation theory strongly depend on the choice of such control functions. 
Table 1. The coefficients $a_{n}$ of the asymptotic expansion for $c_{1}(x)$.

\begin{tabular}{|l|c|c|c|c|c|}
\hline$N$ & 0 & 1 & 2 & 3 & 4 \\
\hline$a_{1}$ & 0.111643 & 0.111643 & 0.111643 & 0.111643 & 0.111643 \\
\hline$a_{2}$ & -0.0264412 & -0.0198309 & -0.0165258 & -0.0145427 & -0.0132206 \\
\hline$a_{3}$ & 0.0086215 & 0.00480687 & 0.00330574 & 0.00253504 & 0.0020754 \\
\hline$a_{4}$ & -0.0034786 & -0.00143209 & -0.000807353 & -0.000536123 & -0.000392939 \\
\hline$a_{5}$ & 0.00164029 & 0.00049561 & 0.000227835 & 0.000130398 & 0.0000852025 \\
\hline
\end{tabular}

Table 2. Self-similar factor approximants $f_{k}^{*}(\infty), k=3,4,5$, and $q_{m}^{*}, m=2,3$.

\begin{tabular}{|c|c|c|c|c|c|}
\hline$N$ & 0 & 1 & 2 & 3 & 4 \\
\hline$f_{3}^{*}$ & 0.548162 & 0.700831 & 0.823464 & 0.92360 & 1.00681 \\
\hline$f_{4}^{*}$ & 0.646479 & 0.858742 & 1.02476 & 1.15706 & 1.26426 \\
\hline$f_{5}^{*}$ & 0.682986 & 0.888388 & 1.05111 & 1.18101 & 1.28629 \\
\hline$q_{2}^{*}$ & 0.7347 & 1.008 & 1.219 & 1.384 & 1.516 \\
\hline$q_{3}^{*}$ & 0.7999 & 1.112 & 1.352 & 1.540 & 1.688 \\
\hline
\end{tabular}

Table 3. Coefficients of polynomials $q(t)=b_{0}+b_{1} t+b_{2} t^{2}$, and self-similar approximants $q^{*}(t)=b_{0}(1+A t)^{n}$.

\begin{tabular}{|c|c|c|c|c|c|}
\hline$N$ & 0 & 1 & 2 & 3 & 4 \\
\hline$b_{0}$ & 0.548162 & 0.700831 & 0.823464 & 0.9236 & 1.00681 \\
\hline$b_{1}$ & 0.129222 & 0.222044 & 0.288772 & 0.338205 & 0.375156 \\
\hline$b_{2}$ & -0.030905 & -0.0641325 & -0.087474 & -0.104749 & -0.11771 \\
\hline$A$ & 0.714061 & 0.894486 & 0.956514 & 0.985622 & 1.00014 \\
\hline$n$ & 0.330135 & 0.354202 & 0.366623 & 0.371523 & 0.372564 \\
\hline
\end{tabular}

\section{Discussion}

We have suggested a method of extrapolating asymptotic series for a small variable $x \rightarrow 0$ to any finite values of $x$, including the infinite limit $x \rightarrow \infty$. The method is based on self-similar approximation theory. We have applied the method to calculating the critical temperature of weakly interacting $O(N)$ symmetric field theory. We have considered the cases with the number of the components $N=0,1,2,3,4$, for which the seven-loop expansions are known. Note that the case of $N=\infty$ allows for an exact solution $[10,40]$ yielding

$$
c_{1}(N=\infty)=\frac{8 \pi}{3[\zeta(3 / 2)]^{4 / 3}}=2.328473 .
$$

As is evident from (11) and (12), knowing $c_{1}$ immediately gives the critical temperature

$$
T_{c} \simeq T_{0}\left(1+c_{1} \gamma\right)
$$

Our results are in very good agreement with Monte Carlo simulations. The advantage of the used self-similar factor approximants is in their simplicity and unique definition, involving no control functions in the final calculations.

We are grateful to B. Kastening for discussions. Financial support from RFBR (grant \#14 - $02-00723$ ) is acknowledged. 
Table 4. Results for the coefficient $c_{1}$ of the critical temperature shift obtained using self-similar factor approximants, compared to Monte Carlo simulations and optimized perturbation theory.

\begin{tabular}{|c|c|c|c|}
\hline$N$ & $c_{1}$ & Monte Carlo & OPT \\
\hline 0 & $0.77 \pm 0.03$ & & $0.81 \pm 0.09$ \\
\hline 1 & $1.06 \pm 0.05$ & $1.09 \pm 0.09[39]$ & $1.07 \pm 0.10$ \\
\hline 2 & $1.29 \pm 0.07$ & $\begin{array}{c}1.29 \pm 0.05[36] \\
1.32 \pm 0.02[37,38]\end{array}$ & $1.27 \pm 0.11$ \\
& & & $1.43 \pm 0.11$ \\
\hline 3 & $1.46 \pm 0.08$ & & $1.54 \pm 0.11$ \\
\hline 4 & $1.60 \pm 0.09$ & $1.60 \pm 0.10[39]$ & \\
\hline
\end{tabular}

\section{References}

[1] H. Satz, Phys. Rep. 88, 349 (1982)

[2] R. Hagedorn, Riv. Nuovo Cimento 6, 1 (1983)

[3] J. Cleymans, R. Gavai, and E. Suhonen, Phys. Rep. 130, 217 (1986)

[4] H. Reeves, Phys. Rep. 201, 335 (1991)

[5] V.I. Yukalov and E.P. Yukalova, Physica A 243, 382 (1997)

[6] V.I. Yukalov and E.P. Yukalova, Phys. Part. Nucl. 28, 37 (1997)

[7] V.I. Yukalov and E.P. Yukalova, POS (Baldin ISHEPP XXI) 2012, 046 (2012)

[8] H. Kleinert, V. Schulde-Frohlinde, Critical Properties of $\phi^{4}$ Theories (World Scientific, Singapore, 2001)

[9] G. Baym, J.P. Blaizot, M. Holzmann, F. Laloë, D. Vautherin, Phys. Rev. Lett. 83, 1703 (1999)

[10] G. Baym, J.P. Balzot, J. Zinn-Justin, Eur. Phys. Lett. 49, 150 (2000)

[11] M. Holzmann, G. Baym, J.P. Balizot, F. Laloë, Phys. Rev. Lett. 87, 120413 (2001)

[12] J. Zinn-Justin, Quantum Field Theory and Critical Phenomena (Clarendon, Oxford, 2002)

[13] J.O. Andersen, Rev. Mod. Phys. 76, 599 (2004)

[14] V.I. Yukalov, Laser Phys. Lett. 1, 435 (2004)

[15] H. Kleinert, Path Integrals (World Scientific, Singapore, 2003)

[16] B. Kastening, Laser Phys. 14, 586 (2004)

[17] B. Kastening, Phys. Rev. A 69, 043613 (2004)

[18] B. Kastening, Phys. Rev. A 70, 043621 (2004)

[19] V.I. Yukalov, Moscow Univ. Phys. Bull. 31, 10 (1976)

[20] V.I. Yukalov, Theor. Math. Phys. 28, 652 (1976)

[21] F.F. de Souza Cruz, M.B. Pinto, R.O. Ramos, Phys. Rev. B 64, 014515 (2001)

[22] F.F. de Souza Cruz, M.B. Pinto, R.O. Ramos, P. Senna, Phys. Rev. A 65, 053613 (2002)

[23] J.L. Kneur, M.B. Pinto, R.O. Ramos, Phys. Rev. A 68, 043615 (2003)

[24] J.L. Kneur, A. Neveu, M.B. Pinto, Phys. Rev. A 69, 053624 (2004)

[25] V.I. Yukalov, Physica A 167, 833 (1990)

[26] V.I. Yukalov, Phys. Rev. A 42, 3324 (1990)

[27] V.I. Yukalov, J. Math. Phys. 32, 1235 (1991)

[28] V.I. Yukalov, J. Math. Phys. 33, 3994 (1992)

[29] V.I. Yukalov and E.P. Yukalova, Ann. Phys. (N.Y.) 277, 219 (1999)

[30] V.I. Yukalov and E.P. Yukalova, Chaos Solit. Fract. 14, 839 (2002)

[31] V.I. Yukalov and E.P. Yukalova, POS (Baldin ISHEPP XXII) 2014, 080 (2014) 
[32] V.I. Yukalov, S. Gluzman, and D. Sornette, Physica A 328, 409 (2003)

[33] S. Gluzman, V.I. Yukalov, and D. Sornette, Phys. Rev. E 67, 026109 (2003)

[34] V.I. Yukalov and E.P. Yukalova, Phys. Lett. A 368, 341 (2007)

[35] V.I. Yukalov and E.P. Yukalova, Eur. Phys. J. B 55, 93 (2007)

[36] V.A. Kashurnikov, N. Prokofev, and B. Svistunov, Phys. Rev. Lett. 87, 120402 (2001)

[37] P. Arnold and G. Moore, Phys. Rev. Lett. 87, 120401 (2001)

[38] P. Arnold and G. Moore, Phys. Rev. E 64, 066113 (2001)

[39] X. Sun, Phys. Rev. E 67, 066702 (2003)

[40] P. Arnold and B. Tomašik, Phys. Rev. A 62, 063604 (2000) 